\title{
From Silicon Chips to Carbon Nanotubes
}

\author{
Mona Nabil Elgohary \\ Faculty of Computers and Information \\ Helwan University \\ Hala Abdelgalil, PhD \\ Faculty of Computers and Information \\ Helwan University
}

\author{
Mostafa-Sami M. Mostafa, PhD \\ Professor \\ Faculty of Computers and Information \\ Helwan University \\ Wessam Elbehaidy, PhD \\ Faculty of Computers and Information \\ Helwan University
}

\begin{abstract}
Nanotechnology is the science that deals with material in the nanoscale dimension. One of the most interesting nanomaterials today is Carbon Nanotubes. This paper contains two parts; the first part summarizes Nanotechnology and Nanoscience by illustrating the history, the technology concepts, the application areas and the commercial products, while the second part explains Carbon Nanotubes; its formation, classes, properties, and its nowadays potential uses.
\end{abstract}

\section{General Terms}

Nanotechnology, Nanomaterial

\section{Keywords}

Nanotechnology, Nanoscience, Carbon Nanotubes,

Nanometer, Nanoscale Dimension, Nanoscale Transistor

\section{INTRODUCTION}

Nanoscience and Nanotechnology are the future of engineering, materials, and related sciences.

"Nano" means very small, so nanomaterials can be constructed atom-by-atom; with different techniques (ex: a bottom-up technique). The information about the bottom-up process is embedded in the material building blocks so that these can self-assemble in the resulting product.

Also, at the nanometer scale, the properties of the material, such as its electrical conductivity, its weight, its color, and its strength, change. The consequence is that a material when in a bulk form; big pieces of materials found around us can have properties which are very different from that nano-sized form. At nanoscale dimension, the principles of classical physics don't don't can describe their behavior (their movement, their energy). At this dimension, the quantum mechanics principles are used. For instance, bulk silver is non-toxic, while Nano silver is toxic and can kill viruses upon contact.

Furthermore,. Nanomaterials have an increased surface -tovolume ratio compared to bulk materials. All processes that occur at the material surface, such as catalysis and detection. The surface-to-volume ratio is significant [8].

\section{FROM NANOSCIENCE TO NANOTECHNOLOGY}

The Nanoscience is commonly defined as: "the study of phenomena and manipulation of materials at atomic, molecular and macromolecular scales, where properties differ significantly from those at a larger scale" [8].
So, the same material (e.g., gold) at the nano-scale (nano gold) can have different electrical, optical, and magnetism properties (even opposite too!) from the properties the material has at the macro scale (bulk).

Nanoscience is considered as "multidisciplinary science", which means that it combines concepts of many sciences such as biology, biotechnology, and engineering. Therefore, Nanoscience is a "horizontal-integrating science that cuts across all vertical sciences and engineering disciplines" as shown in figure $1[8]$.

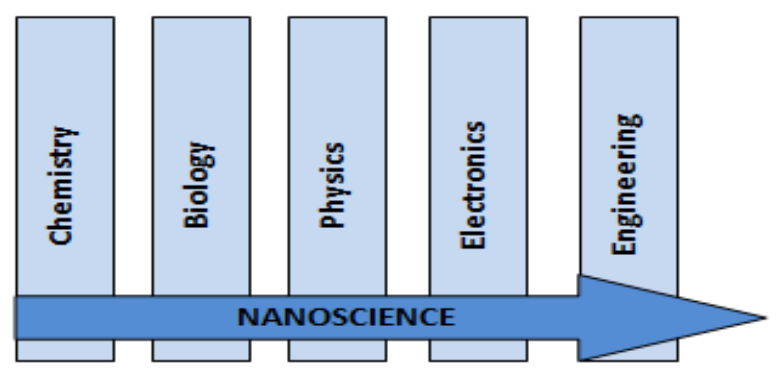

Fig 1: Nanoscience Disciplines

Nanotechnology is the application of Nanoscience in commercial products and many industries (figure 2).

\section{NANOTECHNOLOGY}

\subsection{Introduction}

The Nanotechnologies is commonly defined as: "the design, characterization, production, and application of structures, devices, and systems by controlling shape and size at the nanometer scale." [8].

As shown in figure 2, Nanotechnology cuts "horizontally" across many industrial areas and "enables" the platform and the tools to realize certain products and because it brings together previously separated sectors of science, it is "convergent" [8].

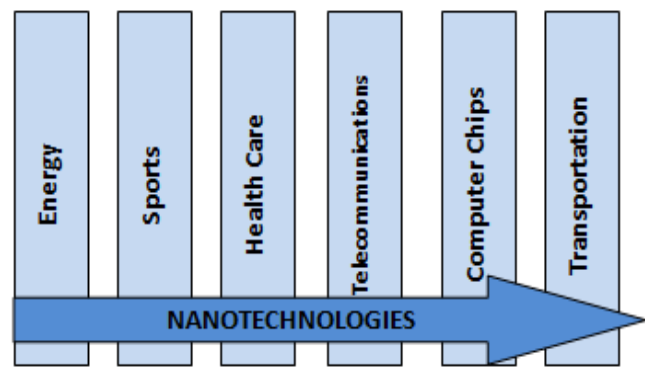

Fig 2: Nanotechnology 


\subsection{Nanotechnology History}

The idea of nanotechnology was firstly highlighted in 1959 when the Nobel Prize winner, Richard Feynman, said, "There is plenty of room at the bottom" in his talk at the Californian Institute of Technology at the meeting of the American physics society. He expected that it is possible to control materials at a very small scale. So, new areas of scientific research would be introduced.

In 1974, nanotechnology term was first suggested by Norio Tangichi from Tokyo University of Science, while the main details of this technology were explored by Eric Drexler 's book "Engines of Creation - The Coming Era of Nanotechnology" in 1986.

Since 1980's, key discoveries of new nanotechnology-enabled materials, nanomaterials (see table1), resulted in developments in many fields including quantum dots, "buckyballs" (i.e. fullerenes or carbon nanotubes), and a range of other new materials and additional applications.

Many of those nanomaterials can support many areas of applications for improving already available products and processes or designing completely new ones.

Fullerenes are a good example; it is a new class of carbon material at the nanoscale, called nanocarbon, with the new chemical, electrical and physical properties. They are very strong mechanically but also flexible and consider as an excellent conductor. It can be used in many applications, like solar cells, various coatings, sports equipment, memory chips etc. [2].

\section{NANOTECHNOLOGY CONCEPTS}

\subsection{Nanometer}

One billionth of a meter $(10-9 \mathrm{~m})$ is one nanometer. The nanometer scale (as shown in figure 3 ) is defined as 1 to 100 $\mathrm{nm}$. The range is set normally to be minimum $1 \mathrm{~nm}$ to avoid single atoms or small group of atoms being designated as nano-objects. So, Nanoscience and nanotechnologies deal with groups of atoms of minimum $1 \mathrm{~nm}$ size.

The nanometer's upper limit is normally $100 \mathrm{~nm}$ but it is not an accurate limit because objects with greater size (up to $1000 \mathrm{~nm}$ ) are defined also as nanomaterials [8].

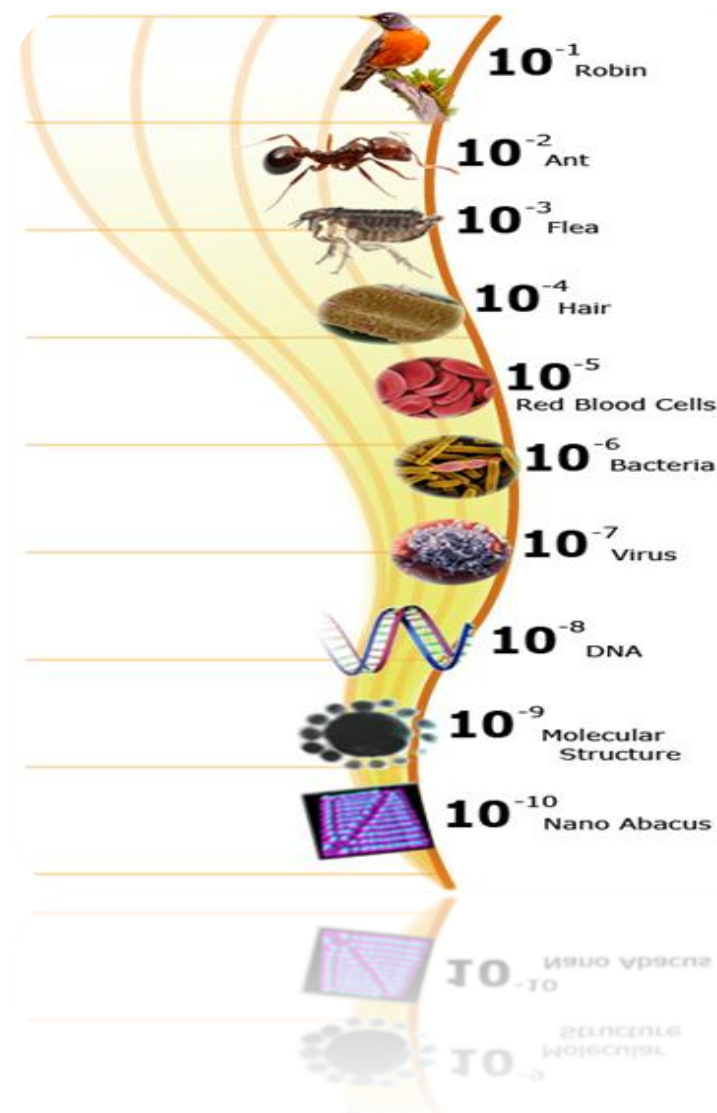

Fig 3: Nanometer

\subsection{Nanomaterial}

A nanomaterial object is an object that has at least one dimension on the nanometer scale (approximately 1-100nm) .According to their dimensions, nanomaterials are classified into 3 groups as shown in table 2 [8].

TABLE 2: Nanomaterial groups

\begin{tabular}{|l|l|l|}
\hline $\begin{array}{l}\text { Nanomaterial } \\
\text { Dimension }\end{array}$ & Example \\
\hline $\begin{array}{l}\text { All three dimensions }< \\
100 \mathrm{~nm}\end{array}$ & $\begin{array}{l}\text { Nanoparticles, Quantum } \\
\text { dots, nanoshells, nanorings, } \\
\text { microcapsules }\end{array}$ & \\
\hline $\begin{array}{l}\text { Two dimensions }<100 \\
\mathrm{~nm}\end{array}$ & $\begin{array}{l}\text { Nanotubes, fibres, } \\
\text { nanowires }\end{array}$ & \\
\hline $\begin{array}{l}\text { One dimension }<100 \\
\mathrm{~nm}\end{array}$ & $\begin{array}{l}\text { Thin films, layers and } \\
\text { coatings }\end{array}$ & \\
\hline
\end{tabular}


TABLE 1: Nanotechnology development mileston

\section{Year}

1959

1966

1974

1981

1982

1984

1986

1986

1986

1987

1989

1990

1991

1991

1990s

1999

2000

2001

2002

2002

2004

2005

2006

2006

2007

2008

2008

\section{Development Milestone}

Nobel prize winner in physics Robert Feynman's “There 's plenty of room at the bottom"

Quantum confinement effect discovered by Alan Fowler and colleagues at IBM

"Nanotechnology" concept presented by Norio Taniguchi of the Tokyo University of Science

STM (Scanning Tunneling Microscope) invented by Gerd Binning and Heinrich Rohrer of IBM, US

Quantum dot, laser application proposed by Yasuhiko Arakawa and Hiroyuki Sakaki , the University of Tokyo

Fullerenes found out by Richard Smilley and colleagues of Rice University in the US

AFM “Atomic Force Microscopy” invented by Gerd Binning and colleagues at IBM

Eric Drexler of the MIT, "Engines of Creation: The Coming Era of Nanotechnology“ US publishes.

Foresight Nanotech Institute established as the first one to educate society about the benefits and risks

First commercial STM shipped by Digital Instruments in the US

First commercial AFM shipped by Digital Instruments in the US

The "IBM" logo produced with individual atoms for promotional reasons

Carbon nanotubes discovered by Sumio Ijima of NEC, Japan

AFM used on living cells, stimulated the cross-pollination between nano- and biotechnology

China adds nanotechnology to its S\&T priorities in the 863 National High Technology Program at MOST

Discovery of dip-pen nanolithography by Chad Mirkin at Northwestern University in the US

The Center for Nanotechnologies at the Chinese Academy of Sciences opens in Beijing

National Nanotechnology Initiative launched in the US

Nanotechnology Research Network Centre of Japan established

The European Commission designated nanotechnology a priority area in the Sixth Framework Program

The " 21 st Century Nanotechnology Research and Development Act" in the US providing further funding

The Japanese "Strategic Technology Roadmap" published

The $3^{\text {rd }}$ Science and Technology Basic Plan" launched in Japan

The EU "Roadmaps at 2015 on Nanotechnology Application" published

Russia announces USD 8 billion investment in nanotechnology from 2007-2015

The US “Technology Roadmap for Productive Nanosystems” published

Korean "Nanotechnology Roadmap" published 


\section{NANOTECHNOLOGY APPROACHES}

Nanotechnology has emerged from gathering many disciplines like chemistry, physics, and biology through the joint realization of new chances of research at the nanoscale. There are two used approaches; Figure 4 explains the steps of both top-down approach and bottom-up approach [2].

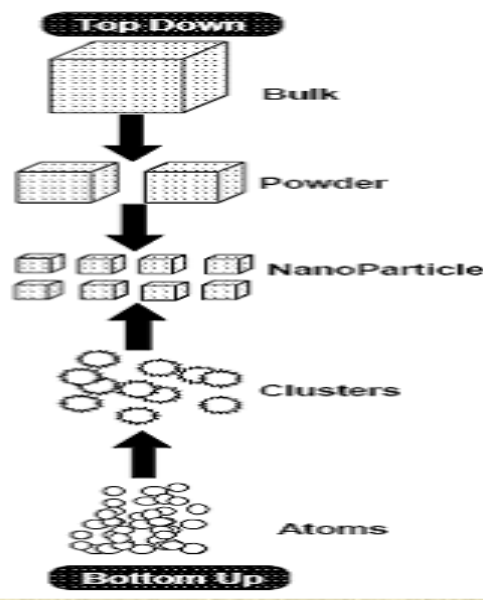

Fig 4: Top-Down and Bottom-Up Approaches

To have a nano particle using top-down approach, the bulk material will have a successive cutting or slicing until reaching nanoscale. On the other hand, "bottom-up" approach refers to construct the material atom by atom, molecule by molecule or cluster by cluster. This builds-up a material from the bottom [11].

\section{NANOTECHNOLOGY APPLICATION AREAS}

\subsection{Drug - Delivery Techniques}

Dendrimers are a type of nanostructure that can be used in a wide range of applications, For example, the treatment of the diseases like cancer. Dendrimers have a great advantage; it can do many things simultaneously: recognizing diseased cells, diagnosing diseased states (including cell death), drug delivery, and reporting outcomes of therapy.

\subsection{Nanofilms}

Nanofilms have many uses nowadays; on computer screens, glasses, and cameras to protect or fix the surfaces. Several nano-elements are used in thin films to have many enhanced features like water resistant, self-washing, anti-reflective, infrared-resistant, anti-microbial, steam-resistant, anti-scratch, and enhance its electrical conductivity[1].

\subsection{Nanoscale Transistors}

Transistors are the electronic gates where a small amount of electric power is used to manage the flow of higher amount of electric power. In computers, providing more transistors will result in a more powerful computer.

With the decrease in transistor size, computers are becoming more powerful. Until recently, a computer chip with the best commercial technology has transistor sizes from 32 nano to 45 nano [1].

\subsection{Water Filtration Techniques}

Carbon nanotubes for water desalination and nanoscale sensors can be widely used to identify pollutants in water networks. There is also titanium dioxide (TiO2) which is a nano-material that has great use for water filtration and water purification [3].

\subsection{Nanotechnology and Space}

Nanotechnology may be the promising technology for making the space-flight. Developments in nanomaterials result in solar sails, shown in figure 5(a) [14], and a wire that can be used for the space elevator, figure 5 (b) [15]. In addition, combining robots and sensors with new nano-materials can enhance the performance of spaceships, spacesuits, and the tools of exploring planets and moons. [5].

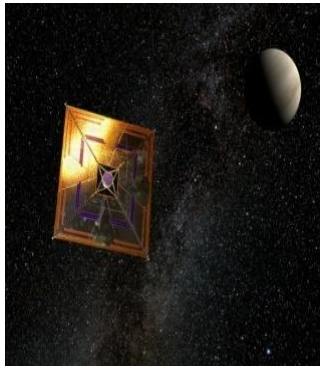

Fig 5: a) Nano Solar Sails

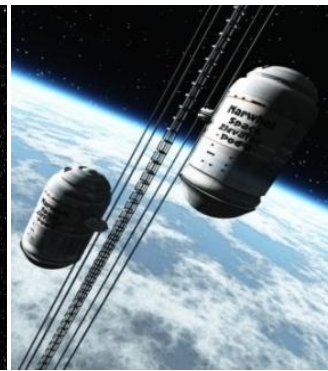

b) Space Elevator

\section{NANOTECHNOLOGY COMMERCIAL PRODUCTS}

\subsection{Anti-Bacterial Products}

One of the most common instances, as shown in figure 6, is the use of silver $(\mathrm{Ag})$ in consumer products as anti-microbial. By combining nanoscale silver into household appliances, textiles, and plastics can kill bacteria without affecting the properties of the products [5].

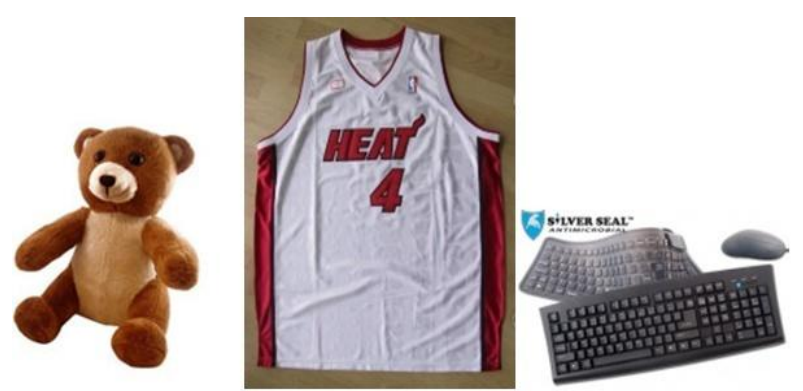

Fig 6: "Benny the Bear" plush toy Sports Jersey from SinotextilesCo,Ltd, Ltd Silver Seal computer keyboard

\subsection{Samsung "Siler Nano" Washers}

It is an advanced washing technology with the capability of the super killing of bacteria .it has 400 billion silver ions dissolved in water to make an extreme cleaning solution that affects the clothes at an almost nano level. 


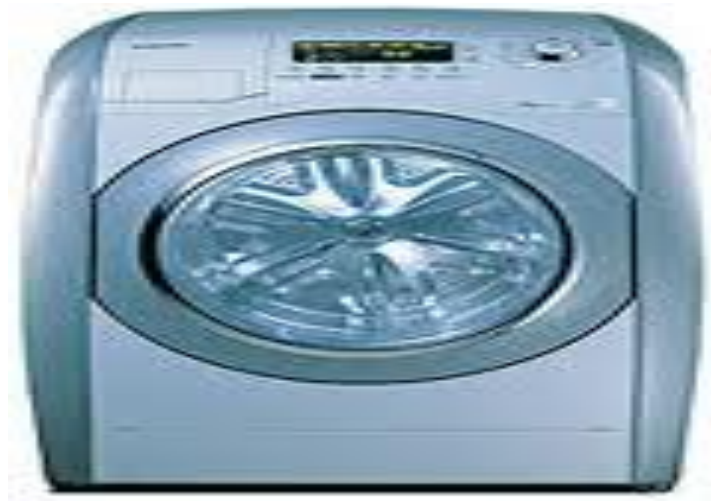

Fig 7: Samsung Silver Nano Washer

Samsung silver nano washer, shown in figure 7 , has a sterilizing the ability to $99.99 \%$ and lasting antibacterial action will redefine the idea of pureness. It uses $99.99 \%$ pure silver for a lasting improvement in your health and garments [7].

\subsection{BMC: lighter and stiffer frames with Easton nanotubes}

Carbon Nanotubes (CNT) technology has powerful possibilities, for instance, this bike frame on the BMC SLC01 Pro Machine that weighs $2.1 \mathrm{lb}$., or about as much as five cell phones. its makers said: " that is Tiny tubes of carbon fiber nanotubes are mixed into the resin which bonds the carbon sheets together, figure 8 , and work to add strength to the resin much like using wire-mesh in concrete does" [6].

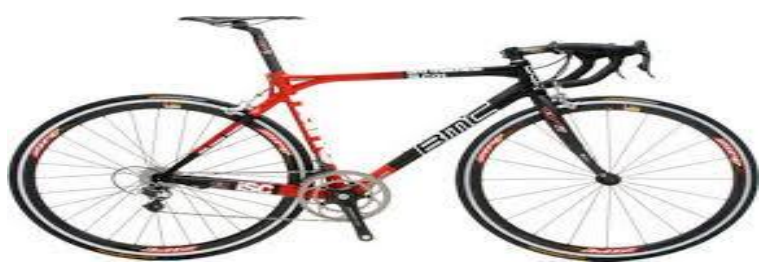

Fig 8: $\quad$ BCM

\subsection{Boeing $787-50 \%$ Composite}

When two or more materials are used together to give a combination of properties this is called composite. Composite materials may be selected to give unusual combination performance, corrosion resistance, conductivity or hardness.

The Boeing 787 makes greater use of composite materials, as shown in figure 9 , in its airframe and primary structure than any previous Boeing commercial airplane by comprising nearly half carbon fiber-reinforced plastic and other composites.

The result is an airplane that offers 20 percent weight savings compared to more traditional aluminum designs [3][4][10][21].
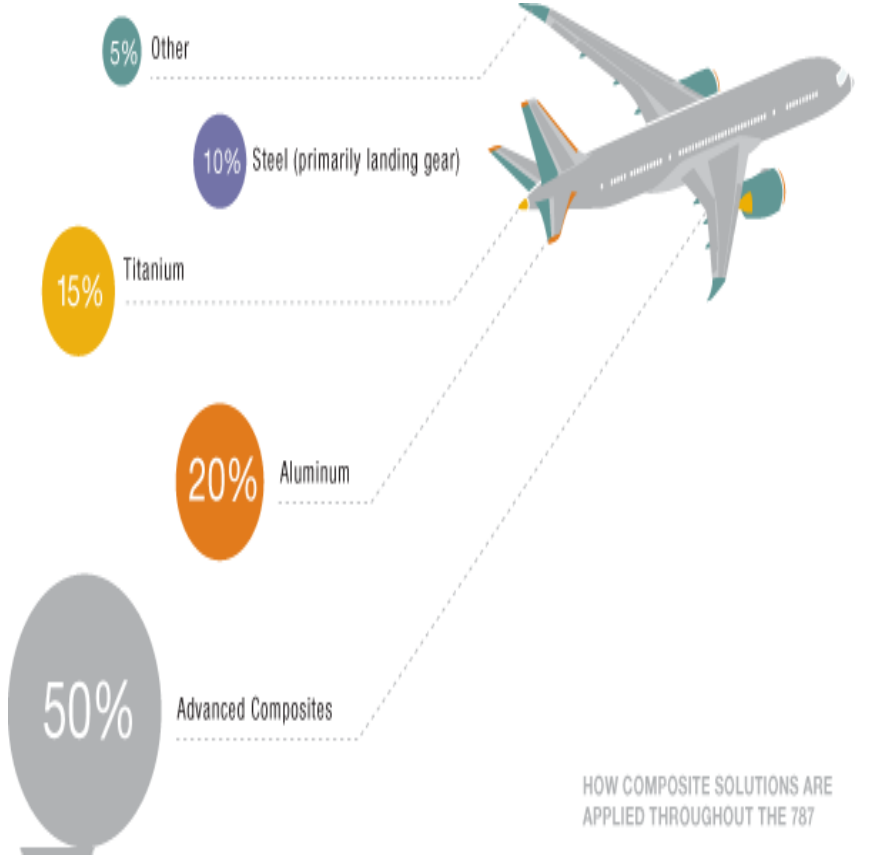

Fig 9: Boeing 787- 50\% Composite

\section{CARBON NANOTUBES}

\subsection{Introduction}

In 1991, Sumio Iijima discovered carbon nanotubes, as in table 1.They are long, thin cylinders of carbon atoms which are novel for their size, shape, and extraordinary physical properties.

Carbon Nanotube is an excellent example of true nanotechnology: it has a very small diameter (less than 100 nanometers and can be as thin as 1 or $2 \mathrm{~nm}$ ). It is a material that can be manipulated chemically and physically in very useful ways. It has important properties, such as extraordinary heat conductivity, electrical conductivity, and mechanical properties, and its high length-to-diameter ratio. These properties make it very useful in a wide range of applications in chemical processing, biology, electronics, energy management, and many other fields to be developed [12].

\subsection{Carbon Nanotubes Formation}

Carbon (C6) is the 4th most available element in the Universe by mass after Hydrogen, Helium, and Oxygen with an atomic number of 6 . It forms more than 10 million organic composites, which makes carbon the chemical basis of all known sort of life [12].

Carbon is an exceptional element which may exist in many stable forms from 3D diamond to $2 \mathrm{D}$ graphene to $1 \mathrm{D}$ nanotubes and $0 \mathrm{D}$ fullerenes (Buckyballs). In figure 10 these forms are shown. [13].

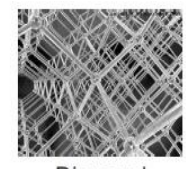

Diamond

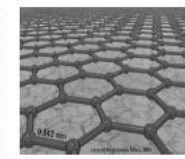

Graphene

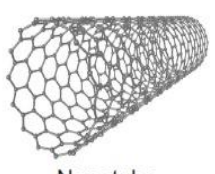

Nanotube

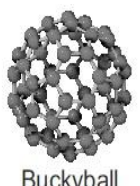

Buckyball
Fig 10: Carbon Forms: 3D Diamond, 2D Graphene, 1D nanotube, 0D Buckyballs 
As shown in figure 11, the carbon nanotube is constructed by some carbon atoms (graphene) that are bonded together in a hexagonal network. Then this graphene rolled into a cylinder and form a carbon nanotube. Based on the physical design of the tubes, their electric, thermal and structural properties can change [12] [13].

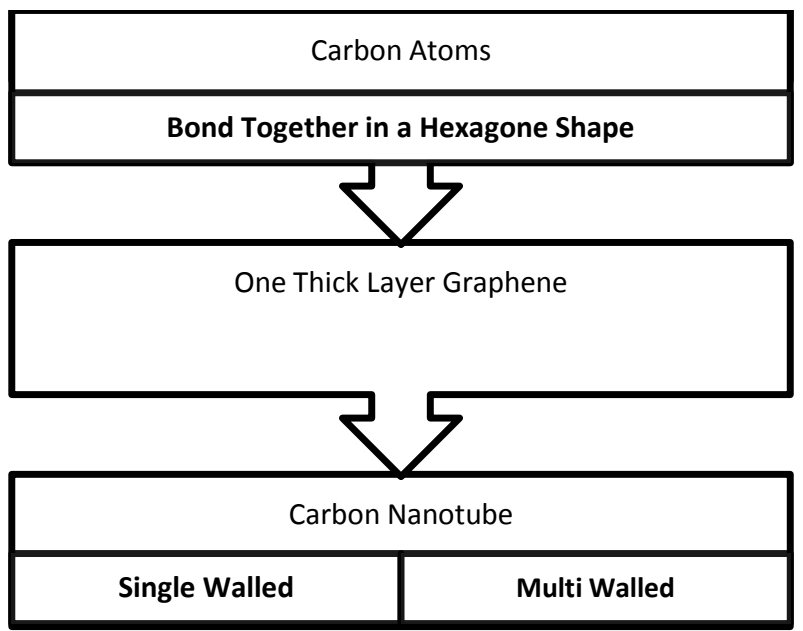

Fig 11: Carbon Nanotubes Formation

\subsection{Carbon Nanotubes and Moore's Law}

According to Moore's Law, by 2019, the transistor's dimension will be just a few molecules. The improvement fro $\mathrm{m}$ a mini, to micro to $45 \mathrm{~nm}$ scale has already known. Carbon Nanotubes have diameters of only 1 to $2 \mathrm{~nm}$, seems to be one of the perfect applicants to deliver us to the end of Moore's Law curve [12].

\subsection{Carbon Nanotubes Classes}

Nanotubes can be Single-Walled Nanotubes (SWNT), or can have Multi-Walled Nanotubes (MWNT) - cylinders inside the other cylinders. The MWNT were the first to be discovered (1991) while SWNT were discovered two years later (1993) [15].

\section{- Single-Walled Carbon Nanotubes}

A single-walled carbon nanotube can be described as a single sheet of carbon atoms (graphene form) which are located on a hexagonal lattice, has a typical diameter of $1-2 \mathrm{~nm}$ rolled up into a seamless hollow cylinder [13].

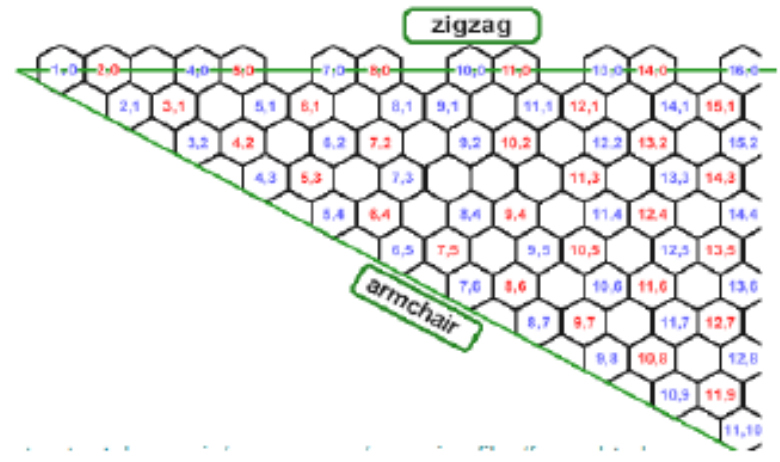

Fig12: Single Walled Carbon Nanotubes (Graphene) Sheet with the possible rolling designs (Zigzag and Armchair)
There are three assorted designs, figure 12 , can be used to form the single walled nanotubes: armchair design, chiral design, and Zigzag design.

The design depends on how the graphene is wrapped into a cylinder. Rolling a sheet of graphene from its corner results in one design. While rolling the graphene from its edge results in another one. Figure 13 shows how the designs of zigzag and armchair are rolled.

The chiral vector) $(n, m)$ represents the design of the nanotubes. [12] [15]. Figure 12 shows that zigzag design is getting when the index $\mathrm{m}$ in rolling vector is constant (i.e. zero) while index $\mathrm{n}$ changes. The indices in Armchair design changes with the same rate, while the indices in chiral design changes differently.

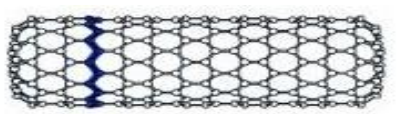

a

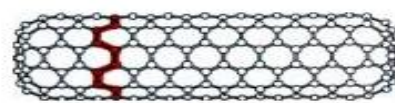

b

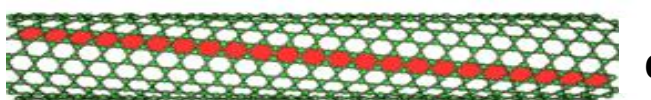

Fig 13: Single Walled Nanotubes Designs according to chiral vector a) (n, 0) Zigzag b) $(\mathbf{n}, \mathbf{n})$ Armchair c) $(\mathbf{n}, \mathbf{m})$ Chiral [9].

Figure 13 is a real atomically resolved image of a chiral nanotube, Figure $12 \mathrm{c}$, as observed in STM (Scanning Tunneling Microscope) experiments. Measuring chirality and the diameter (i.e. height) of the nanotube, one can uniquely characterize the nanotube under study [9]

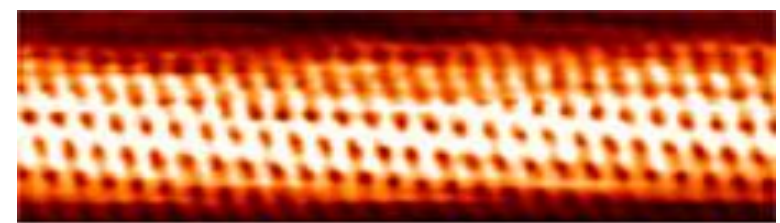

Fig 14: Microscopic Image for Chital SWNT

Nanotube's electrical properties are affected by the structural design. For example, when $(n, m)$ is a multiple of 3 , it has an Armchair design described as "metallic" and it is highly conducting, as figure 15(a). Otherwise, the nanotube is a semiconductor, when it has Zigzag design and Chiral Design, as in figure 15(b) [17] [14]
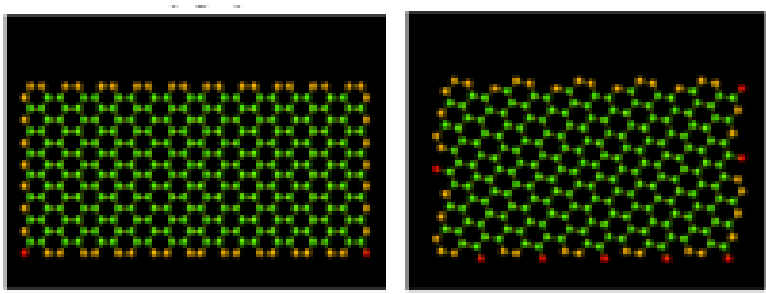

Fig 15: a) Armchair Design

b) Chiral Design Metallic

High Conductivity
Semiconductor 
- Multi-walled carbon nanotubes (MWNT) Parchment model and Russian Doll model are two structural models of multi-walled nanotubes.

Figure 16 shows Parchment model where a single sheet of carbon is rolled around itself more than one times.

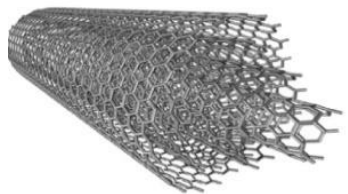

Fig: 16 Parchment Model for MWNT

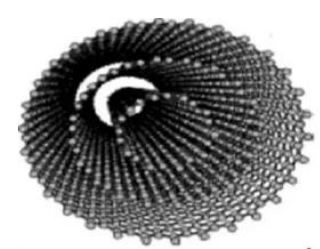

Fig 17: Russian Model for MWNT

Russian Doll model, figure 17, a carbon nanotube contains another nanotube; the inner tube has a smaller diameter than the outer one.

Multi-walled carbon nanotubes have same properties as single-walled nanotubes but in multi-walled nanotubes, the outer wall protects the inner one from interacting chemically with outside materials. Furthermore, multi-walled nanotubes also have a higher tensile strength than single nanotubes [13].

\subsection{Carbon Nanotubes Properties}

\section{- $\quad$ Strength}

There are $\mathrm{sp} 2$ bonds between the individual carbon molecules, and this bond is even stronger than the sp3 diamond's bond. This means the tensile strength of carbon nanotubes have a higher than steel and Kevlar. Individual carbon tubes can bond together under high pressure, converting some $\mathrm{sp}^{3}$ bonds to $\mathrm{sp}^{2}$. This means producing a nano-wire becomes possible.

Carbon nanotubes are not only strong but also they are flexible. You can press on the tip of a nanotube and cause it to bend without breaking the tube and it will return to its original shape when the pressure is removed. [12].

Based on this property, there are many uses of nanotubes like waterproof and tear resistant cloth fabrics, concrete and steel like applications (a space elevator)

\section{Electrical Properties}

As mentioned in figure 15(a, b), carbon nanotube's design effects its conductivity power. Nanotubes become high conductive when the formation of carbon atoms reduces the collisions between electrons and atoms.

Carbon nanotubes also have the ability to carry higher electric currents than copper because of their $\mathrm{sp} 2$ bonds between carbon atoms.
Furthermore, when carbon nanotubes are used for interconnection of semi-conducting devices it can transfer electrical signals at speeds up to $10 \mathrm{GHz}$ [12].

Based on this property, there are many uses of nanotubes like electrical circuits

\section{- $\quad$ Thermal Properties}

The strength of $\mathrm{sp} 2$ bonds between the carbon atoms enables them to withstand high temperatures. So, nanotubes can be used as thermal conductors and transfer more than 15 times the amount of watts per meter per Kelvin compared to the commonly used copper wire. Based on the tubes' temperature and its environment, the nanotube's thermal conductivity may change [12].

Based on this property, there are many uses of nanotubes like: sensors, vacuum proof food packaging.

\subsection{Carbon Nanotubes Potential Uses}

Here are some uses of Carbon Nanotubes:

\section{- Batteries}

Nowadays, "Rechargeable Lithium-Ion Batteries" are the most used batteries for most portable electronic devices. These batteries contain two electrodes, the first one is graphite and the second is the metal oxide. When lithium ions move between these two electrodes, the batteries release charge.

At the "University of North Carolina", [12, 15] the researcher have proved that the storage capacity can imply doubled by replacing the graphite with singlewalled carbon nanotubes.

Carbon nanotubes electrodes have many advantages; they can be ten times lighter and thinner than carbon electrodes and they have a conductivity power that is more than one thousand times greater.

In some cases like electric vehicles, the reduction in weight can make a great reduction in power requirements of the battery.

Carbon nanotubes are used in supercapacitors producing a power cycle of $30 \mathrm{kw} / \mathrm{kg}$ while the best commercial devices result in only $4 \mathrm{kw} / \mathrm{kg}$. The best usage of these nano- capacitors is in laptops and cell phones because these capacitors take less time to recharge devices.

\section{- Solar Cells}

Researchers at the Georgia Institute of Technology [12] used carbon nanotubes implanted on iron-coated silicon chips to build towers with a height of $200 \mu \mathrm{m}$. This tower is a group of thousands of carbon nanotubes with vertical alignment, and these towers are the main part of nanotubes new solar cells. Single square centimeter, $\mathrm{cm} 2$, of a single solar cell's surface contains forty thousand, 40,000 of these towers.

These cells absorb more light and then reflect it towards the sides of the towers. And also the nano-particles solar cells have two peaks at 45 degrees and operate with relatively high efficiency during most of the day. While the traditional solar cells that have peak efficiency only when the sun is at 90 degrees. This makes nanotube cells particularly suitable for space applications because there is no mechanical way to change the angle to face the sun. 


\section{- Nano-Electronics}

The most important areas of possible applications where single-walled nanotubes can be used are nano-electronics because nanotubes have high electrical conductivity. CNT can also be considered the most famous conductive carbon fibers.

Alternative designs for carbon nanotubes can lead to semiconducting materials, such as silicon.

Based on the degree of volatility - the degree of growth and size of nanotubes - conductivity in nanotubes may be very high making nanotubes the best suitable element for interconnection between electronic circuits

\section{- Interconnect}

Microchip industries need metal compounds to act as the main transistor connections on fine chips. Initial designs used aluminum to connect transistors, then change to copper.

A few years later, copper technology will be inefficient to connect circuits because there are some expectations that high-performance chips that are connected to more tightly packed transistors will need interconnections with $40 \mathrm{~nm}$ or less in width, which can be achieved using carbon nanotubes.

Toshiba and Stanford University[12,13] recently announced results proving that the operation of a nanotube-based interconnects at $1 \mathrm{Ghz}$ on a chip carrying 11000 transistors on a microchip the size of $1 / 100$ th of a square inch[12,16].

\section{REFERENCES}

[1] Debnath Bhattacharyya, Shashank Singh, Niraj Satnalika, Ankesh Khandelwal, and Seung-Hwan Jeon, "Nanotechnology, Big things from a Tiny World: a Review", International Journal of u- and eService, Science and Technology, Vol. 2, No. 3, September 2009.

[2] Christopher Palmberg, Hélène Dernis and Claire Miguet, "NANOTECHNOLOGY: AN OVERVIEW BASED ON INDICATORS AND STATISTICS”, DSTI/DOC, 2007.

[3] Michael Krause, MSPH, ROH, CIH, "Introduction toNanotechnology", VERITOX technology and industrial hygiene, 2010

[4] Donald R. Askeland, Pradeep. P. Fulay "Essentials of Materials Science and Engineering", Third Edition, January 2017.

[5] UW MRSEC Education Group, "What is Nanotechnology",http://education.mrsec.wisc.edu/3 5.htm, December 2016.

[6] Charlie White, "Bike Frame Uses Nanotube Technology, Light as Five Cell phones", http://gizmodo.com/162457/bike-frame-usesnanotube-technology-light-as-five-cellphones , 2006
[7] Samsung Group, "Samsung SilverNano Washing Machine",http://www.samsung.com/in/support/skp/ faq/433965, December 2016

[8] Luisa Filipponi and Duncan Sutherland, "Chapter 1 Introduction to Nanoscience and Nanotechnologies", Interdisciplinary Nanoscience Center (iNANO), Aarhus University, Denmark, September 2010

[9] Nanotube Research Team, "Types of Nanotubes “, http://www.photon.t.utokyo.ac.jp/ maruyama/kataura/chirality.html

[10] "Boeing 787 from the Ground Up", http://www.boeing.com/commercial/aeromaga zine/articles/qtr_4_06/article_04_2.html , 2011

[11] Sri D.Suneel, "Role of Bottom-up and Top-Down approaches in Nanotechnology", http://eresources.gitam.edu/nano/NANOTECHNOL OGY/role_of_bottomup_and_topdown_a.htm

[12] [12] Chris Scoville, Robin Cole, Jason Hogg, Omar Farooque, and Archie Russell, "Carbon Nanotubes"

[13] Sami SAPMAZ, "Carbon nanotube quantum dots", 2006

[14] Kannan Balasubramanian and Marko Burghard "Chemically Functionalized Carbon Nanotubes", small journal , 2005

[15] "Potential Applications of Carbon Nanotubes “,https://en.wikipedia.org/wiki/Potential_application s_of_carbon_nanotubes

[16] Ali Eatemadi , Hadis Daraee , Hamzeh Karimkhanloo , Mohammad Kouhi, Nosratollah Zarghami, Abolfazl Akbarzadeh, Mozhgan Abasi , Younes Hanifehpour and Sang Woo Joo , "Carbon nanotubes: properties, synthesis, purification, and medical applications", Nanoscale Research Letters a Springer Open journal , 2014.

[17] "Dendritic polymers for smart drug delivery applications",http://pubs.rsc.org/en/Content/Article Html/2015/NR/c5nr90030a , July 2015.

[18] Nanofilm",https://www.bioohio.com/item/nanofilm/ , November 2013

[19] "Sustainable Nano", http://sustainable nano.com/2016/02/16/nanomaterials-in-bicyclespart-1/, February 2016.

[20] “ReclassifyingNanosilver",https://pubs.acs.org/cen/ news/84/i49/8449notw7.html , 2006.

[21] Boeing's

Dreamliner,https://www.thoughtco.com/boeing787-dreamliner-820385, Todd Johnson, 02 April 2017 